\title{
EL CONTROL GUBERNAMENTAL Y LAS AMENAZAS DISRUPTIVAS EN ECUADOR
}

GOVERNMENT CONTROL AND DISRUPTIVE THREATS IN ECUADOR

\author{
Carlos Luis Redroban Ortiz*
}

\begin{abstract}
Resumen
Disrupción es una frase que nos pone en alerta por su traducción del término inglés disruption en "ruptura brusca"; el hackeo de un servidor y la exposición de información sensible de los ecuatorianos al mundo podría mal conceptualizarse como esa ruptura brusca en el ámbito digital, sin embargo, dicho hackeo, realmente expuso una debilidad del sistema de control interno, en el cual se evidenció que el único que no tiene acceso libre a toda la data que se genera en Ecuador es el auditor gubernamental. El presente artículo parte con el relato de un caso para análisis, presenta un marco teórico relacionado al control interno, legislación y tecnologías disruptivas, expone las debilidades del control interno gubernamental desde el enfoque de un profesional de auditoría del sector público y concluye con los desafíos que representan las innovaciones disruptivas en los procesos técnicos de auditorías en pos de conseguir la eficiencia en el control de la administración pública.
\end{abstract}

Palabras Clave: Auditoría gubernamental; Control gubernamental; Control interno gubernamental; Disrupción digital; Hackeo a data pública, Tecnología disruptiva.

\begin{abstract}
Disruption is a phrase that alerts us due to its translation of the English term disruption into "abrupt rupture"; The hacking of a server and the exposure of sensitive information of Ecuadorians to the world could be misconceived as that abrupt break in the digital sphere, however, said hack actually exposed a weakness of the internal control system, in which it was evidenced that the only one who does not have free access to all the information generated in Ecuador is the government auditor. This article starts with a case report for analysis, presents a theoretical framework related to internal control, legislation and disruptive technologies, exposes the weaknesses of government internal control from the perspective of a public sector audit professional and concludes with the challenges that represent disruptive innovations in the technical auditing processes in pursuit of achieving efficiency in the control of public administration.
\end{abstract}

Keywords: Government audit; Government control; Government internal control; Digital disruption; Hacking public data, Disruptive technology

Recibido: 19 de octubre de 2021 /Aceptado: 17 de enero de 2022

\footnotetext{
* Ingeniero en Ciencias Empresariales. Abogado. Magíster en Administración de Empresas. Magíster en Supply Chain Management, PhD (c). Gerente de Auditoría Interna Gubernamental, Corporación Financiera Nacional CFN B.P. Docente posgrado Universidad UTEG y docente Universidad ECOTEC, Ecuador, correo: credrobano@ecotec.edu.ec; credroban@uca.edu.ar; ORCID: https://orcid.org/0000-0002-0672-5323
} 


\section{Introducción}

En el contexto de la administración pública, el avance vertiginoso de la tecnología y el desarrollo de destrezas en el manejo de la información ha permitido la evolución de los sistemas de gestión pública para atender de forma eficaz y eficiente las necesidades que demandad los ciudadanos de una nación, sin embargo en el ámbito del control gubernamental, se evidencia que dichas tecnologías y destrezas no están del todo presentes en las entidades estatales y que son fácilmente vulnerables ante las amenazas de hackers que identifican las falencias de control en la administración de la información, y que paradójicamente pueden acceder a la data que un auditor gubernamental aspira obtener en un proceso de auditoría, pero que sin embargo es casi imposible hacerlo por las limitaciones legales, tecnológicas, presupuestarias y de conocimientos. La filtración de datos que sufrió Ecuador en el año 2019, puso de manifiesto esta hipótesis en tiempos en que la innovación disruptiva y las filosofías políticas contraria de ser un aliado para un auditor gubernamental se ha convertido en una amenaza en nuestros países latinoamericanos.

\section{Experiencia Pública}

\section{1 Filtración de datos de Ecuador}

Ecuador, es un país sudamericano, que según el Instituto Nacional de Estadísticas y Censos (INEC), al 21 de octubre de 2019, cuenta con una población de 17’345.856 habitantes, con un gobierno democrático elegido cada cuatro años, es el único país en la región con un sistema dolarizado, cuenta con veinte y cuatro provincias distribuidas en la costa, sierra, oriente y región insular y por su diversidad ecológica es considerado como uno de los países con mayor biodiversidad del mundo que alberga a ciudadanos del mundo los cuales por razones familiares, laborales, económicas y sociales tienen su residencia habitual en este territorio.

El 16 de septiembre de 2019, la BBC publicó una noticia grave para los intereses de todos los ciudadanos nacionales y extranjeros que viven en Ecuador titulada "Filtración de datos en Ecuador: la 'grave falla informática' que expuso la información personal de casi toda la población del país sudamericano"; noticia alarmante, pues se expuso información sensible que se encontraba administrada por la empresa ecuatoriana de consultoría Novaestrat que se dedica al análisis de datos, marketing y desarrollo de software.

Según la compañía de seguridad informática VpnMentor (2019), que trabaja bajo la modalidad de hacking ético, "un servidor utilizado por una empresa de análisis de datos y que contenía información personal sobre millones de ecuatorianos no contaba con los protocolos de protección necesarios y por lo tanto, casi cualquier persona podía acceder a ellos", constituyéndose esta situación como la mayor filtración en línea de información personal en la historia del país sudamericano y una de las mayores en Latinoamérica, dado el número de personas expuestas (BBC, 2019).

Los datos confidenciales expuestos incluyeron nombres completos, fecha de nacimiento, lugar de nacimiento, estado civil, direcciones, números de teléfono, correos electrónicos, información sobre miembros de la familia, información financiera, etc., tanto de personas naturales como de personas jurídicas, como resultado del hackeo del servidor de la empresa Novaestrat que contenía información de las siguientes instituciones públicas: Servicio de Rentas Internas (SRI), Registro Civil, Instituto Ecuatoriano de Seguridad Social (IESS), Banco del Instituto Ecuatoriano de Seguridad Social (BIESS), Secretaría de Educación Superior Ciencia y Tecnología (SENESCYT) y la Agencia Nacional de Tránsito (ANT). 


\subsection{Control gubernamental en Ecuador.}

El pueblo es el mandante y primer fiscalizador del poder público, en ejercicio de su derecho a la participación establece el artículo 204 de la Constitución de la República del Ecuador, en consecuencia le otorga el derecho a todo ciudadano a denunciar cualquier acto que se considere en perjuicio del Estado, de igual forma la carta magna ecuatoriana en su artículo 211 establece que "La Contraloría General del Estado es un organismo técnico encargado del control de la utilización de los recursos estatales, y la consecución de los objetivos de las instituciones del Estado y de las personas jurídicas de derecho privado que dispongan de recursos públicos", en consecuencia le otorga la facultad y funciones de: 1. Dirigir el sistema de control administrativo ... .- 2. Determinar responsabilidades administrativas y civiles culposas e indicios de responsabilidad penal, ... .- 3. Expedir la normativa para el cumplimiento de sus funciones.- 4. Asesorar a los órganos y entidades del Estado cuando se le solicite.

En consecuencia, el control gubernamental ecuatoriano es competencia de la Contraloría General del Estado, entidad estatal que tiene como misión "Controlar los recursos públicos para precautelar su uso eficiente, en beneficio de la sociedad" y cuya visión es "Ser el organismo innovador en la prevención y control del uso de los recursos públicos, generando valor y beneficio para la ciudadanía", observando el debido proceso y aplicando metodologías técnicas previamente normadas (COSO), cumple su función de control de la gestión pública por medio de auditorías a las entidades estatales y privadas que administran recursos públicos concluyendo finalmente con recomendaciones que se plasman en los respectivos informes.

En este proceso de control, el auditor gubernamental debe cumplir con una serie de procedimientos con miras a contrastar la información evidenciada en los hallazgos de tal manera que el contenido de los informes sea verás y concluyente.

Para conseguirlo debe sortear una serie de trámites burocráticos para llegar a obtener dicha información; dentro de este procedimiento juega un papel muy importante las entidades que generan y administran información que en mucho de los casos las guarda bajo el principio de reserva y confidencialidad, por la sensibilidad de los datos empero que lastimosamente como se señaló inicialmente fue expuesta, rompiéndose dichos principios y evidenciando una falencia en el sistema de control informático en nuestro país.

El legislador ecuatoriano ha emitido leyes con miras a tratar de ir acorde a los avances y nuevas tecnologías tales como:

- Ley de Comercio Electrónico, Firmas y Mensajes de Datos que "regula los mensajes de datos, la firma electrónica, los servicios de certificación, la contratación electrónica y telemática, la prestación de servicios electrónicos, a través de redes de información, incluido el comercio electrónico y la protección a los usuarios de estos sistemas";

- Código Orgánico Administrativo que "regula el ejercicio de la función administrativa de los organismos que conforman el sector público";

- Ley para la Optimización y Eficiencia de Trámites Administrativos que dispone "la optimización de trámites administrativos, regular su simplificación y reducir sus costos de gestión, con el fin de facilitar la relación entre las y los administrados y la Administración Pública y entre las entidades que la componen; así como, garantizar el derecho de las personas a contar con una Administración Pública eficiente, eficaz, transparente y de calidad"; cambiar la cultura burocrática resulta más complicado en la práctica pues se sigue exigiendo y dependiendo de la generación de documentación física más aún para el servicio público, lo que conlleva a que el trabajo del auditor gubernamental de igual manera caiga en ese círculo burocrático que ralentiza su trabajo, pese a que paradójicamente la misma:

- Ley Orgánica de la Contraloría General del Estado en la que se establece que para efectos de auditorías "el auditor gubernamental tendrá acceso irrestricto a registros, 
archivos y demás documentos que sustenten la información, y cuando la naturaleza de

la auditoría lo requiera, a las operaciones en sí".

Complementariamente a estas leyes, los informes de auditoría gubernamental se convierten en documentos que evidencien el accionar de los servidores públicos, sancionan sus malas prácticas y contienen recomendaciones que son de cumplimiento obligatorio para los directivos de las instituciones auditadas; el contenido de dichos informes deben constituirse en instrumentos de consulta y guía para los servidores públicos que ven en la Contraloría General del Estado a parte de una entidad fiscalizadora y de control, una entidad que los guíe, asesore y pre alerte de cometer errores involuntarios que por acción u omisión puedan devenir en sanciones administrativas, civiles y penales posteriores.

El organismo de control gubernamental ecuatoriano no trabaja aislada del mundo del control, pues regionalmente es parte de las Entidades Fiscalizadoras Superiores de los países del MERCOSUR y Asociados (EFSUR) conformado por: Argentina, Bolivia, Brasil, Chile, Colombia, Ecuador, Paraguay, Perú, Uruguay y Venezuela; y, desde el año 2012, tiene el carácter de Grupo Subregional dentro de la Organización de Entidades Fiscalizadoras Superiores de Latinoamérica y el Caribe (OLACEFS), que es el organismo internacional, autónomo, independiente, apolítico y de carácter permanente que tiene por objetivo fomentar el intercambio experiencias y buenas prácticas relacionadas a la fiscalización y al control gubernamental, así como al fomento de las relaciones de cooperación y desarrollo profesional entre sus miembros y partes interesadas. OLACEFS es parte de la Organización Internacional de Entidades Fiscalizadoras Superiores (INTOSAI) que es la organización central para la fiscalización pública exterior. Es una organización no gubernamental con un estatus especial con el Consejo Económico y Social de las Naciones Unidas.

\subsection{Irrupción e incendio de edificio de la Contraloría General del Estado.}

Producto de medidas económicas tomadas por el gobierno de turno en octubre de 2019; en Ecuador se comenzó a revivir el escenario de protestas que eran comunes en la década del 80 y 90, cuando los gobiernos electos democráticamente no concluían sus períodos electorales y el pueblo se encargaba de derrocarlos por malas decisiones políticas. Esta vez fue el Decreto Presidencial 883 de 1 de octubre de 2019, (Luque, Moreno y Zubizarreta, 2020), que originó el inicio de protestas masivas en todo el territorio ecuatoriano, en dicho documento se contempló la eliminación del subsidio de combustibles expresamente el diésel y la gasolina extra de mayor consumo a nivel nacional.

Pese a que el objetivo de las protestas siempre ha sido, el Palacio de Carondelet (sede del ejecutivo y residencia oficial del Presidente de la República) en la capital ecuatoriana - Quito, por tradición los grupos sociales y políticos principalmente indígenas, se han agrupado en el sitio denominado "el arbolito", diagonal a las instalaciones de la Asamblea Nacional y la Contraloría General del Estado.

El país por varias décadas ha soportado manifestaciones y los grupos se han reunidos siempre en el mismo sitio "el arbolito", sin embargo en esta ocasión por primera vez el edificio matriz de la Contraloría General del Estado sufrió una primera irrupción a sus instalaciones el 7 de octubre perpetrando un conato de saqueo y posteriormente el 12 de octubre de 2019, el edificio fue atacado por segunda ocasión por un grupo de manifestantes que procedieron a incendiar el edificio y destruir lo que encontraban a su paso.

Esta situación mereció el repudio del gobierno, las autoridades, la comunidad nacional y comunidad internacional precisamente en una época donde la máxima institución de control público del Estado maneja varios procesos en el que están relacionadas altas autoridades de los gobiernos pasados y en estas instalaciones se archiva toda la documentación e información relacionada a las auditorías gubernamentales. 
La situación desarrollada en Quito - Ecuador en octubre de 2019, puso en evidencia una vez más la vulnerabilidad de la Contraloría General del Estado ante un riesgo latente que el tiempo se ha encargado de evidenciarlo, representada en esta ocasión por las hordas de protestantes que dependiendo de quien las lidere se convierten en una gran amenaza para impedir que esta entidad como máxima institución de control público cumpla con su función constitucional de velar por el correcto uso de los recursos estatales (Lavell, 2008).

Pese a que gran cantidad de archivos físicos de la Contraloría General del Estado fueron destruidos y el incendio afectó una gran extensión de su infraestructura física; gracias a los sistemas informáticos, a la aplicación de la legislación vigente, al liderazgo situacional y nivel de compromiso institucional; en poco tiempo sus sistemas administrativos e informáticos comenzaban a operar y estabilizarse permitiendo la operatividad del sistema de control gubernamental en red con todas las provincias del país y la recuperación de los archivos en formato digital.

\subsection{Disrupción digital}

La gestión de la tecnología de la información, en alineación al concepto de disrupción digital no solo permite encontrar valor en los modelos de negocio, sino que además visualiza y enfrenta los cambios tecnológicos basándose en las necesidades de los clientes cada vez más exigentes e informados, todo ello en aplicación de la ética y la responsabilidad en beneficio de la sociedad (Varon, D. Frasica, D. Gamboa, E. Benavides D. y Vargas H. 2017).

McKelvey y MacDonald (2019) exponen un ejemplo de participación ciudadana aplicando la disrupción digital que realizó el gobierno canadiense cuando en el 2016 experimentó un proceso de consulta muy abierto durante el desarrollo de su autorregulación de inteligencia artificial utilizando herramientas gratuitas de google, estos autores al citar a (Bingham, Nabatchi, \& O'Leary, 2005)., señalan que a pesar de que la consulta estaba lejos de ser inclusiva, el ejercicio intentó buscar nuevas formas de involucrar al público en general en el proceso de desarrollo de políticas públicas, parte de un nuevo énfasis en la prestación de servicios digitales en el gobierno federal y un giro prometedor hacia nuevos modelos de consulta a los ciudadanos en materia de política pública en general, conflictos y cambios políticos y sociales.

Por otra parte, el Tribunal de Cuentas de Holanda decidió crear un laboratorio de innovación. Su objetivo era estimular, apoyar y difundir la innovación en su organización por medio de la participación activa de auditores y ciudadanos para juntos proponer soluciones a los problemas de la mala gestión pública; así mismo vio en el sistema de información geográfica SIG (sistema para elaboración de planos) que permite a los usuarios almacenar y mantener una gran cantidad de información relacionada con datos geográficos para visualizar y simplificar datos complejos, para crear nuevos datos de datos existentes y para producir mapas de alta calidad, una herramienta útil para las diferentes fases de una auditoría, evaluación de los riesgos relevantes, diseño de la auditoría, realización de la auditoría, análisis de los resultados de la auditoría y comunicación de los resultados de la auditoría (Stuiveling, 2015).

Hay muchos ejemplos de disrupciones que se desarrollaron lentamente y, por lo general, involucran «el auge» de algo: de Internet en la década de 1990, de China como potencia económica en la década de 2000, de la computación en la nube en la década de 2010.

Los auditores gubernamentales pueden elegir entre varias disrupciones posibles que se encuentran disponibles actualmente y que surgen de ejemplos como: los automóviles autónomos, la inteligencia artificial (IA), la Internet de los Objetos, las políticas de comercio proteccionistas y, muy probablemente, muchas más (IIA, 2018). 


\subsection{Tecnologías disruptivas en la auditoría}

Deloitte, 2018 con la siguiente gráfica muestra "los avances en ciencia de datos capacidades de procesamiento, y las más nuevas tecnologías que han provocado la $4^{\text {a }}$ Revolución Industria, cambiando nuestro mundo hacia lo digital”.

Los avances tecnológicos y las tendencias en analíticas avanzadas, automatización robótica de procesos [robotic process automation (RPA)], e inteligencia cognitiva [cognitive intelligence $(\mathrm{CI})$ ] rápidamente están remodelando los modelos de negocio, mejorando la productividad, y permitiendo la innovación en la manera como las organizaciones conectan productos y servicios para sus consumidores Deloitte (2018).

A medida que los humanos y las máquinas colaboran más estrechamente, y las innovaciones de inteligencia artificial salen del laboratorio de investigación y se incorporan a las actividades empresariales tanto públicas como privadas entre las que se puede destacar la gestión del control, las posibilidades de transformación son asombrosas PwC (2017).

Carolyn Saint, directora ejecutiva de auditoría de la Universidad de Virginia afirma "Un desafío enorme y real es cómo explicar las repercusiones de una disrupción que está por venir a los líderes políticos, de forma tal que la organización pueda responder a las necesidades de la ciudadanía de una forma diferente". El gran desafío para los auditores en la actualidad es percibir las disrupciones en su verdadera forma, reconocer lo que está por venir y proporcionarle una perspectiva al ente de control sobre cómo aprovechar el factor disruptivo para el control de los recursos públicos, eso es realmente valioso. No hay nada nuevo en el proceso técnico de auditoría que se resumen en la contrastación de lo actuado versus lo que se debía hacer conforme lo establece la norma, bajo el principio heteronómico que regula a la función pública. (IIA, 2018).

Ilustración 1: Deloitte, Industry 4.0: Challenges and Solutions for the Digital Transformation of Exponential Technologies, Deloitte AG, 2015 and Deloitte proprietary research

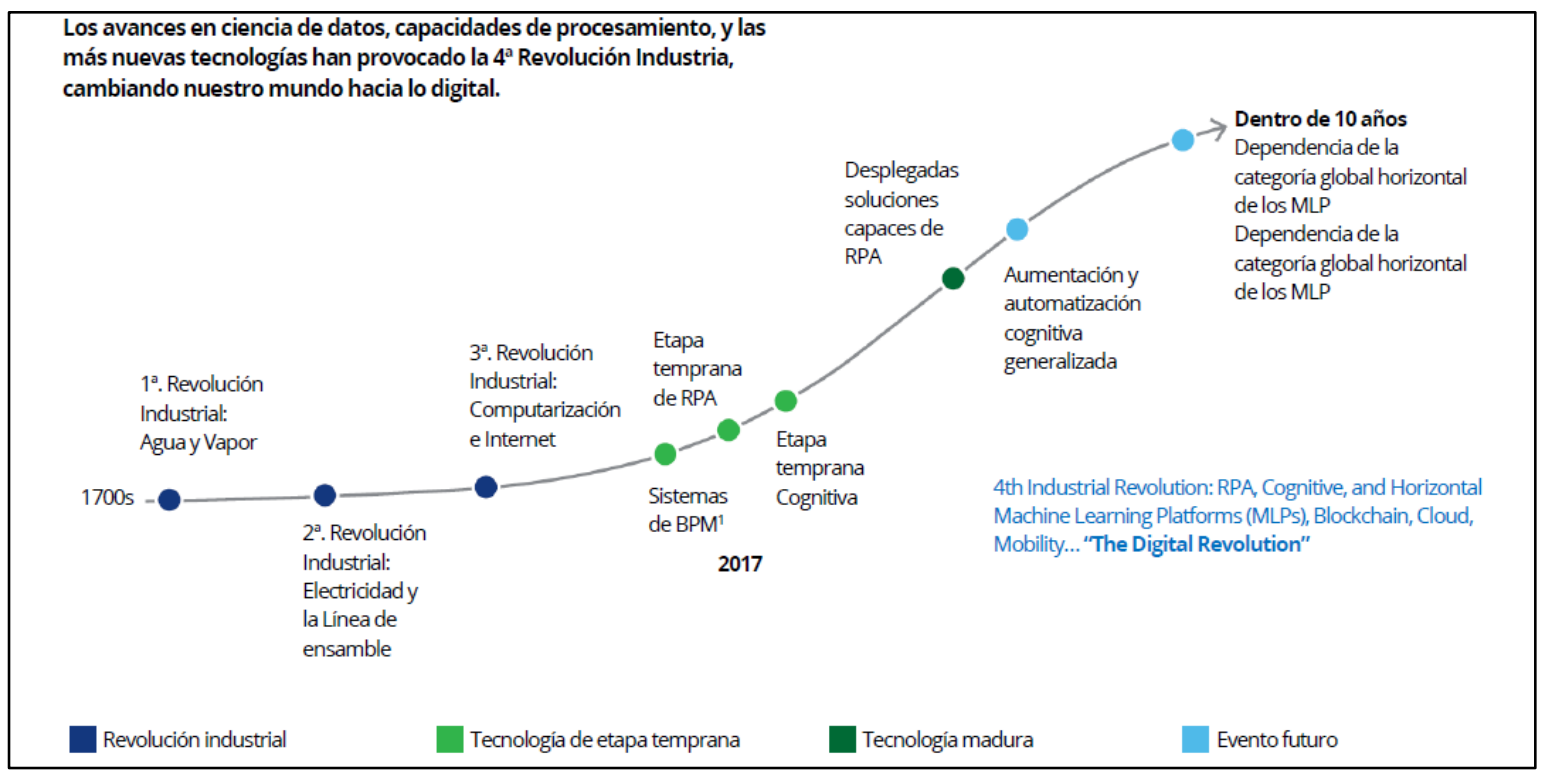

Fuente: Deloitte (2018)

La era en que la información se generaba de forma impresa o manual, se usaba, se gestionaba y finalmente se eliminaba o archivaba, procesos que facilitaba de cierta forma el trabajo del auditor que creaba o reconstruía las pistas de auditoría siguiendo el rastro de papel prácticamente quedó relegada con la introducción de la información electrónica, que hicieron 
las cosas mucho más complejas. Hoy en día, la información de diversas fuentes y con diversas funciones fluye al mismo tiempo y desde el momento en que se genera, es inmediatamente utilizada, reutilizada, modificada, almacenada o eliminada (Stuiveling, 2015).

En la encuesta "Estado de Auditoría Interna" de PwC (2017), un 68 por ciento de los miembros de los consejos de administración y un 77 por ciento de la dirección creen que la función de auditoría interna no está haciendo lo suficiente para ayudar a gestionar la disrupción, más de la mitad cree que a las unidades de auditoría les falta conocimientos en la materia para gestionar la disrupción; un 38 por ciento identifica como la causa principal a la falta de recursos financieros, factor principal sobre todo en la administración pública y entes de control gubernamental que limitan sus alcances de auditoría por falta de presupuesto. (IIA, 2018)

The Institute of Internal Auditors, Inc. (2018), afirma que "hay varias formas en las cuales los auditores internos pueden ayudar a gestionar el efecto de las tecnologías disruptivas en sus organizaciones", ésta guía también es aplicable a las actividades de control gubernamental que realizan las instituciones fiscalizadoras de la gestión pública y que se resume en lo siguiente:

Concentrarse en el aseguramiento. - La auditoría debería continuar concentrándose en lo que mejor hace. Al continuar enfocándose en la gestión de riesgos, el control y el gobierno, los auditores pueden ayudar a asegurar que los procesos estén diseñados y funcionen de forma eficaz (independientemente de la velocidad de la disrupción). Ayudar proactivamente a la organización a prever los riesgos emergentes y cambios tecnológicos puede posicionar a auditoría interna como una autoridad, y esta puede ayudar a preparar a la organización para responder a los eventos disruptivos.

Trabajar con las partes interesadas y los expertos en la materia.- Alinear el trabajo de auditoría con las expectativas de las partes interesadas clave de auditoría. Trabajar de cerca con expertos en la materia que estén implementando tecnologías disruptivas y concentrarse en los temas más relevantes y significativos.

Invertir en la formación sobre tecnologías disruptivas.- Dedicarse constantemente a capacitarse para aprender sobre las nuevas tecnologías y los nuevos riesgos complejos y emergentes que se incorporan en las organizaciones. Los máximos directivos de auditoría deberían desarrollar un modelo de dotación de personal innovador, flexible y adaptable para sacar partido de una reserva de talentos altamente especializados que tenga la competencia tecnológica y la habilidad de comprender y aprovechar rápidamente las nuevas herramientas, técnicas y procesos.

Poner a trabajar las nuevas tecnologías.- Adoptar y aprovechar las nuevas tecnologías a la hora de realizar el trabajo de auditoría. Los auditores deben estar a la vanguardia y adoptar la inteligencia artificial, la informática cognitiva y los robots inteligentes. Los auditores deben comprender cómo funcionan las tecnologías como la cadena de bloques y cómo se pueden usar en sus organizaciones. Deben sacar provecho del aprendizaje automático y del análisis de datos en sus procesos de auditoría (la auditoría en tiempo real debería ser un requisito a medida que las organizaciones implementan nuevos procesos operativos o administrativos). (IIA, 2018, p.6)

El servicio de auditoría no puede apartarse de esta realidad sobre todo la gubernamental pues se trata de aprovechar las tecnologías disruptivas para controlar de mejor manera los recursos públicos; este avance vertiginoso demanda un cambio radical en el sistema de control que va desde la cultura organizacional, adaptación de legislación, redireccionamiento estratégico y reperfilamiento de los auditores gubernamentales para acoplarse a los nuevos sistemas y metodologías.

Muchos factores han intervenido para que los profesionales de auditoría gubernamental inventen y reinventen constantemente procesos que permitan por una parte llegar a determinar el correcto uso de los recursos públicos y por otra parte identificar a los malos funcionarios 
públicos que administran dolosa o erróneamente el erario de una nación; empero, si en este siglo se esperaba llegar a la quinta revolución industrial con la puesta en marcha de la red 5G, que permitiría potenciar los procesos de auditoría, no cabe duda que la pandemia que atraviesa el mundo entero debido al Covid-19, ha acelerado el proceso de innovar en muchos campos del quehacer social, económico, educativo, político, familiar, tecnológico pero sobre todo en el ámbito del control gubernamental.

La aparición del internet en el siglo pasado definitivamente cambió los procesos innovadores, pero erróneamente se piensa que la innovación es directamente proporcional a la existencia de la red y que sin ella es imposible innovar. No cabe duda de que el internet facilita los procesos innovadores pero la esencia de la innovación está en el ser humano por ello la importancia de dar al auditor gubernamental las herramientas y la capacitación necesaria para aprovechar las innovaciones y tecnologías disruptivas para el cumplimiento eficiente y eficaz de las tareas asignadas en cada acción de control.

Entidades fiscalizadoras de la EFSUR, OLACEFS e INTOSAI como la Contraloría General de Chile lideran programas impulsados por la organización para la Cooperación y el Desarrollo Económico (OCDE), relacionados con la innovación tecnológica para fortalecer el control gubernamental en época de pandemia y pos pandemia, en las que resalta la importancia de desarrollar nuevas competencias en los auditores gubernamentales, motivar la participación de la ciudadanía en los procesos de control, democratizar la información, reestructurar procesos de auditoría e invertir en infraestructura tecnológica adecuada; de ahí la importancia del trabajo coordinado de las entidades fiscalizadoras regionales y mundiales para replicar las buenas prácticas de control en cada uno de sus países (Nieto y Domínguez, 2016). 


\section{Conclusiones}

Los hechos narrados confirman la hipótesis, pues si bien es cierto la auditoría gubernamental es un proceso sistémico con miras a transparentar la gestión pública, no es menos cierto que al igual que cualquier empresa que brinda servicios, basan su gestión en procedimientos, cumplen con normativas, se apoyan en recursos humanos, financieros y tecnológicos y están en constante búsqueda de innovaciones para mantenerse competitiva en el mercado. pero no basta solo con introducir innovaciones en los procesos sino en redefinir los enfoques estratégicos (Christensen, Raynor y McDonald, 2015).

Carlo, J. Lyytinen, K. y Rose, G. (2011), señalaban que "la secuencia predominante de adopción inicial de las innovaciones disruptivas de las tecnologías de la información se da primero como innovaciones básicas, seguidas de innovaciones de servicios y finalmente por innovaciones de procesos".

Se ha demostrado que sólo aquellas organizaciones que han sabido adecuar su capacidad de cambio a través del uso intensivo del conocimiento, la tecnología, el desarrollo de capacidad de absorción tecnológica y la asimilación y generación de innovaciones han podido crecer al mantener altos estándares de competitividad (Roja, 2016).

Sin importar cuál sea el tipo de administración estatal, la influencia política que se ejerza sobre ésta o el nivel de madurez de la entidad con relación a la adopción de la digitalización disruptiva, es imperativo que los entes de control gubernamental y sus departamentos de auditoría anticipen y alineen los esfuerzos para monitorear riesgos emergentes, desarrollar estrategias, e implementar estrategias de remediación del riesgo. Las analíticas y las nuevas tecnologías les permiten a los departamentos de auditoría desarrollar perspectivas perspicaces, proactivas, y centradas-en-el-futuro Deloitte (2018).

Saskia J. Stuiveling, Presidenta del Tribunal de Cuentas de Holanda, en el año 2015 presentó un informe sobre las nuevas tendencias en la auditoría gubernamental e hizo una reflexión sobre la necesidad de innovación constante en el sector público (incluyendo a los auditores) y resaltó la importancia de buscar los medios para lograr acercar las auditorías a los ciudadanos.

La Contraloría General de una nación como organización de control no puede apartarse del hecho que debe también estar en constante actualización tanto de procesos, normativas, tecnologías de la información, estructura organizacional, etc., pues su función constitucional, le exige la continua y constante interrelación con todos los organismos y funcionarios públicos que a la vez se interrelacionan entre si y entre las entidades, lo que conlleva a ser parte activa de ese entorno exigente y altamente cambiante.

Al iniciar una auditoría en una entidad estatal, sus miembros que por el tiempo de prestación de servicios en dichas entidades y por el acceso a la información sea de forma directa o indirecta (fuentes, redes sociales) son los que realmente conocen los presuntos hechos, actos u operaciones originados en las instituciones de un Estado, que revelan, por acción u omisión de sus servidores o terceros relacionados con ella, la indebida, ilegal o ineficiente gestión y/o utilización de recursos y bienes del Estado; así como también los actos reñidos con la ética pública cometidos por servidores de la misma entidad de control y fiscalización.

Esa interrelación con ellos y con las demás entidades puede converger en una tecnología disruptiva que permita acceder a la data pública que como ya se señaló inicialmente so pena de tener carácter reservado dificulta el trabajo del auditor gubernamental y dilata los informes por falta de atención oportuna pero que sin embargo fue fácilmente vulnerable con un poco de ingenio, conocimiento, inteligencia y alto nivel de descuido.

Para poder beneficiarse y mejorar la capacidad de aprendizaje de los fiscalizados los auditores gubernamentales deben estar en contacto con la sociedad que está en constante cambio y sufre el desafío de las incertidumbres. Como auditores gubernamentales necesitan la 
capacidad de mirar de una manera nueva y por diferentes vías los fenómenos nuevos, si no lo hacen, están cerrando los ojos a la realidad y éstos tendrán menos impacto para la sociedad a la que deben servir y rendir cuentas como parte activa del servicio público.

Las instituciones públicas tienden a crear nuevas normas y procedimientos, y a introducir nuevas responsabilidades para hacer frente a los cambios y desafíos de la sociedad. Pero la dinámica de la sociedad va más allá de la dinámica del proceso de toma de decisiones dentro del sector público: las reglas son fijas y difíciles de cambiar, mientras que la sociedad está cambiando rápidamente. Los gobiernos, por tanto, tienen dificultades para afrontar los cambios y desafíos de la sociedad y su dinámica, y ésta es una de las razones por las que la brecha entre el gobierno y la sociedad se está haciendo mayor (Stuiveling, 2015).

Ilustración 2: Innovación disruptiva para el control gubernamental. El sueño de aun auditor gubernamental.

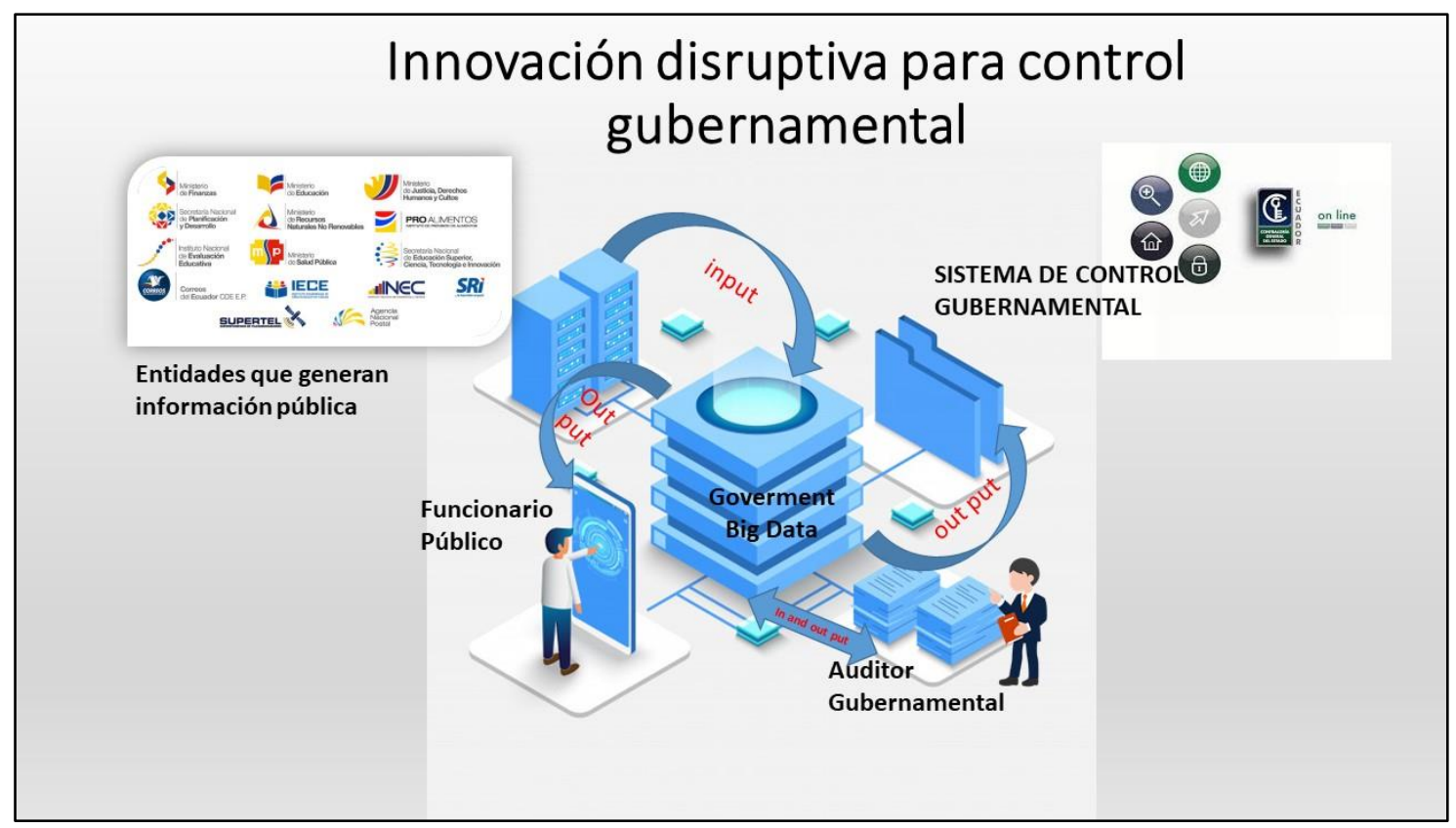

Fuente: Elaboración propia.

Ecuador no está exento de sufrir un nuevo hackeo de la información de sus ciudadanos, ni de que su principal entidad de control de recursos estatales como es la Contraloría General del Estado, sea atacada a futuro o alguna otra entidad pública, pero al igual que un hallazgo de auditoría, no se puede ocultar y se debe mostrar como son, de tal manera que estos hechos sirvan para buscar nuevas alternativas, la innovación disruptiva surge como respuesta a un ambiente de intensos cambios (Varon-Serna et al., 2017) y como tal, el control gubernamental debe adaptarse a ese entorno cambiante para el correcto control de los recursos públicos, a fin de lograr que el out-put sirva no solo para facilitar el trabajo del auditor gubernamental sino que sea parte de un sistema sinérgico que permita interactuar a todos los involucrados en el proceso de auditoría per sé y también los actores de una sociedad.

Las fuerzas que impulsan la disrupción incluyen la tecnología, la política, la demografía $\mathrm{y}$ a veces algunos acontecimientos aleatorios (como protesta social) que sacuden los entornos de las empresas tanto públicas como privadas y calan hasta su propio núcleo. (IIA, 2018).

La innovación significa algo más que el desarrollo de una herramienta o un instrumento: se trata de tener la mente abierta y de no tener miedo de probar cosas nuevas o de hacer las cosas de una manera nueva. Son también innovaciones las intervenciones culturales: alientan 
a las organizaciones a estar al tanto de su cultura, a revisarlas críticamente y a estar dispuestas a cambiarla-rompiendo tabúes-.

Como sostiene Stuiveling (2015), el entorno en que operamos como auditores gubernamentales varía rápidamente, y si no somos capaces de hacer frente a estos cambios para hacer las cosas de otra manera, los resultados serán deficientes y en consecuencia la sociedad nos obligará a cambiar. Pues en calidad de auditores gubernamentales somos el brazo ejecutor del control del recurso público en representación de nuestra sociedad. 


\section{Referencias:}

Asamblea Constituyente (2008). Constitución de la República del Ecuador. Montecristi: Asamblea Constituyente.

Asamblea Nacional República del Ecuador (2017). Código Orgánico Administrativo. Quito: Asamblea Nacional República del Ecuador.

Asamblea Nacional República del Ecuador (2018). Ley para la Optimización y Eficiencia de Trámites Administrativos. Quito: Asamblea Nacional República del Ecuador.

Carlo J., Lyytinen K., Rose G. (2011). Internet computing as a disruptive information technology innovation: The role of strong order effects. Information Systems Journal, 21(1):91-122

Christensen, C. M., Raynor, M. E., \& McDonald, R. (2015). ¿ Qué es la innovación disruptiva. Harvard Business Review, 93(12), 44-53.

Congreso Nacional del Ecuador (2002). Ley de Comercio Electrónico Firmas y Mensajes de Datos. Quito: Congreso Nacional del Ecuador.

Congreso Nacional del Ecuador (2002). Ley Orgánica de la Contraloría General del Estado. Quito: Congreso Nacional del Ecuador.

Deloitte, GG (2018). Auditing the risks of disruptive technologies. Recuperado de https://www2.deloitte.com/content/dam/Deloitte/us/Documents/finance/us-rfa-auditingthe-risks-of-disruptive-technologies.pdf

Lavell, A. (2008). Una Visión de Futuro: La Gestión del Riesgo. Programa Desarrollo Local y Gestión territorial, PNUD. 2-3-6-12-20

Luque, A., Moreno, C. P., \& Zubizarreta, J. H. (2020). Análisis del levantamiento indígena de 2019 en Ecuador: Entre la respuesta legal y el Lawfare. NULLIUS: Revista de pensamiento crítico en el ámbito de Derecho, 1(1), 18-45.

McKelvey, F. y MacDonald, M. (2019). Artificial Intelligence Policy Innovations at the Canadian Federal Government. Canadian Journal of Communication Policy Portal Vol 44 (2019) PP-43-50. 2019

Nieto, E. P., \& Dominguez, F. (2016). OLACEFS. International Journal of Government Auditing.

Ortiz, S. y Silva V. (18 de septiembre de 2019). Bases de datos de seis entidades públicas del Ecuador se filtraron. Diario EL COMERCIO. Recuperado de https://www.elcomercio.com/actualidad/bases-datos-entidades-publicas-filtracion.html

PwC (2017) Sizing the prize. What's the real value of AI for your business and how can you capitalise? Recuperado de https://www.pwc.com/gx/en/issues/data-andanalytics/publications/artificial-intelligence-study.html

Roja H. (2016). The role of knowledge-based firms (KBF) and technology-based firms (TB F) for innovation. Revista Gestión de las Personas y Tecnología, 9:(27):65-80.

Stuiveling, S. (2015). Nuevas tendencias en la auditoría: ¿cómo gestiona la innovación el Tribunal de Cuentas de Holanda?. Revista Española de Control Externo 15 - 33

The Institute of Internal Auditors (2018). Auditoría interna en la era de la disrupción. Recuperado de https://global.theiia.org/knowledge/Pages/Global-Perspectives-andInsights.aspx

Varon Serna D.R., Frasica Rodríguez D.M., Gamboa Andradre E., Benavides Sánchez D. y Vargas Polanco H. (2017). La disrupción digital (I): modelos de negocio, innovación, conocimiento y tecnología. Revista Vector, 12: 22-31.

Vpn Mentor. (16 de septiembre de 2019). Informe: Infracción ecuatoriana revela datos personales confidenc [Mensaje en un blog]. Recuperado de https://www.vpnmentor.com/blog/report-ecuador-leak/ 
Yeung, J. (16 de septiembre de 2019). Filtración de datos en Ecuador: la "grave falla informática" que expuso la información personal de casi toda la población del país sudamericano. BBC. Recuperado de https://www.bbc.com/mundo/noticias-america-latina-49721456 